\title{
A new multichannel broadband NIRS system for quantitative monitoring of brain hemodynamics and metabolism during seizures
}

De Roever, Isabel, Vezyroglou, Aikaterini, Hebden, Peter, Thornton, Rachel, Worley, Alan, et al.

Isabel De Roever, Aikaterini Vezyroglou, Peter Hebden, Rachel Thornton, Alan Worley, Mariana Alves, Emma Dean, Gemma Bale, Paola Pinti, J. Helen Cross, Ilias Tachtsidis, "A new multichannel broadband NIRS system for quantitative monitoring of brain hemodynamics and metabolism during seizures," Proc. SPIE 11074, Diffuse Optical Spectroscopy and Imaging VII, 1107406 (11 July 2019); doi: 10.1117/12.2527067

SPIE. Event: European Conferences on Biomedical Optics, 2019, Munich, Germany 


\title{
A new multichannel broadband NIRS system for quantitative monitoring of brain hemodynamics and metabolism during seizures
}

\author{
Isabel De Roever ${ }^{\mathrm{a}}$, Aikaterini Vezyroglou ${ }^{\mathrm{b}, \mathrm{c}}$, Peter Hebden ${ }^{\mathrm{a}}$, Rachel Thorton ${ }^{\mathrm{c}}$, Alan Worley ${ }^{\mathrm{c}}$, \\ Mariana Alves $^{\mathrm{c}}$, Emma Dean $^{\mathrm{c}}$, Gemma Bale $^{\mathrm{a}}$, Paola Pinti ${ }^{\mathrm{a}}$, J. Helen Cross ${ }^{\mathrm{b}, \mathrm{c}}$, Ilias Tachtsidis ${ }^{\mathrm{a}^{*}}$ \\ ${ }^{a}$ Medical Physics and Biomedical Engineering, University College London, London, UK \\ b Institute of Child Health, Great Ormond Street BRC, University College London, London, UK \\ ${ }^{\mathrm{c}}$ Great Ormond Street Hospital, London, UK
}

\begin{abstract}
We present a newly developed multichannel broadband NIRS (or bNIRS) system that has the capacity to measure changes in light attenuation of 308 NIR wavelengths $(610 \mathrm{~nm}$ to $918 \mathrm{~nm})$ simultaneously over 16 different brain locations. To achieve this the instrument uses a lens based spectrometer with a front-illuminated CCD that has a sensor size of $26.8 \times 26 \mathrm{~mm}$. This large CCD detector allows the simultaneous binning of 16 detector fibres. The software uses the UCLn algorithm to quantify the changes in oxy-, deoxy- haemoglobin concentration $\left(\mathrm{HbO}_{2}, \mathrm{HHb}\right)$ and oxidised cytochrome-coxidase (oxCCO) simultaneously over 16 different brain locations with 1second sampling rate. We demonstrate the use of the instrument in quantifying brain tissue oxygenation and metabolic activity simultaneously with electrical changes as measured with EEG in children with seizures.
\end{abstract}

Keywords: broadband, NIRS, diffuse optics, brain metabolism, cytochrome-c-oxidase, seizures

\section{INTRODUCTION}

The brain is extremely sensitive to imbalances in the supply and demand of oxygen and metabolic substrates. An imaging modality that can reflect the entire scope of cerebral haemodynamics, oxygenation and metabolism at the patient bedside, can improve diagnosis and treatment assessment in brain injury. Early infantile epileptic encephalopathies (EIEE) are progressive childhood disorders characterized by frequent recurring paroxysmal events and epileptic seizures that lead to poor long-term neurological function and development. To elucidate and better understand the pathophysiology of paroxysmal events in EIEE, we need to understand their impact on brain oxygenation and cellular metabolism. Until recently, it has not been possible to measure in-vivo markers of metabolism non-invasively, however, recent advances in broadband near-infrared spectroscopy and imaging (or bNIRS) have made this possible [1]. Mitra and colleagues measured the concentration changes in the oxidation state of cerebral cytochrome-c-oxidase $(\Delta[\mathrm{oxCCO}])$ and hemodynamics during generalized recurrent neonatal seizures following hypoxic-ischemic encephalopathy in a newborn infant using bNIRS [2]. The authors notice that progressive decline in the $\Delta[\mathrm{oxCCO}]$ baseline during seizures suggests a progressive decrease of mitochondrial oxidative metabolism. The aim of this study is to develop a new bNIRS instrument with the capacity to measure at many different locations on the head the brain tissue haemoglobin oxygenation and oxCCO synchronously with EEG providing a unique opportunity to investigate neurovascular and neurometabolic coupling in seizures.

\section{METHODS AND MATERIALS}

The newly developed multichannel bNIRS system has the capacity to measure changes in light attenuation of 308 nearinfrared (NIR) wavelengths (610nm to $918 \mathrm{~nm}$ ) and using the UCLn algorithm quantify the changes in oxy-, deoxyhaemoglobin concentration $\left(\mathrm{HbO}_{2}, \mathrm{HHb}\right)$ and oxCCO simultaneously over 16 different brain locations. The sampling rate of the bNIRS system is $2 \mathrm{~Hz}$. A picture of the system is shown in Figure 1 and represents a significant upgrade to previous instruments by Bale et al (2014) and Phan et al (2016) [3,4].

\subsection{Instrumentation}

The bNIRS imaging system is composed of two broadband tungsten halogen light sources, (HL-2000, Ocean Optics), shown in Figure 1(a), that emit light between 360-2400nm. A 650nm long-pass filter is used to remove shorter wavelengths and hence minimize heat deposition and UV exposure applied to tissue. Light source fibres custom-built by Loptek

*i.tachtsidis@ucl.ac.uk

Diffuse Optical Spectroscopy and Imaging VII, edited by Hamid Dehghani, Heidrun Wabnitz, Proc. of SPIE-OSA Vol. 11074, 1107406 · @ 2019 SPIE-OSA · CCC code: 1605-7422/19/\$21 · doi: 10.1117/12.2527067 
(Germany) were used to transmit the light to the head. The bundle of fibres had a high numerical aperture with a bundle diameter of $2.8 \mathrm{~mm}$ surrounded by an outer casing of $10 \mathrm{~mm}$. Fibres were $2 \mathrm{~m}$ long and connected to the light sources via an SMA connector. Reflected light from the head collected by customized fibres (Loptek) with a numerical aperture of 0.57; this allowed for a minimum detector head height of $5 \mathrm{~mm}$ with fibres still being able to bend 90 degrees from the head surface. Each detector fibre consists of a fibre bundle with diameter of $1 \mathrm{~mm}$ and a casing of $5 \mathrm{~mm}$. The sixteen detector fibres are bundled vertically into a ferrule with a $25 \mathrm{~mm}$ diameter, with each fibre bundle separated by $0.5 \mathrm{~mm}$ casing (see Figure 1(b)). The Acton Series LS785 spectrometer (Princeton Instruments) was used to separate the incoming light into its wavelength components. It is a lens-based spectrograph enabling a higher throughput than mirror-based systems with fast $\mathrm{f} / 2$ lenses enabling direct fibre optic coupling. Anti-reflective coated lenses provide optimum NIR transmission (between 750-1050nm) of over 99\% transmission throughout the working range of the spectrograph. The diffraction grating inside the spectrometer has a bandwidth of $308 \mathrm{~nm}$, with a wavelength range between $610 \mathrm{~nm}$ and $918 \mathrm{~nm}$. The grating is blazed at $750 \mathrm{~nm}$ and has 600 grooves $/ \mathrm{mm}$. After light has been diffracted at the grating, it is focused (with an $\mathrm{f} / 2$ lens) onto a charge-coupled device (CCD) the PIXIS 1300F (Princeton Instruments). It is a front-illuminated CCD with a two-dimensional imaging array, with a sensor size of $26.8 \times 26 \mathrm{~mm}$. This sensor contains a 1340x1300 pixels imaging array, with pixel size is $20 \times 20 \mu \mathrm{m}$.

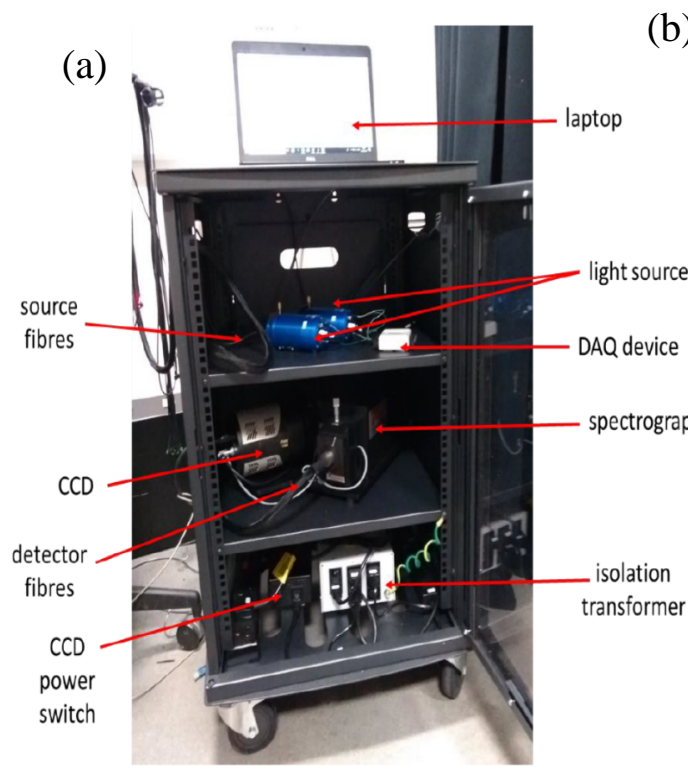

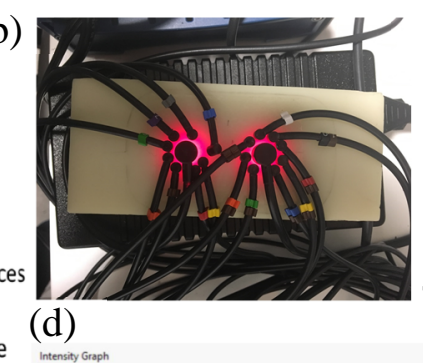

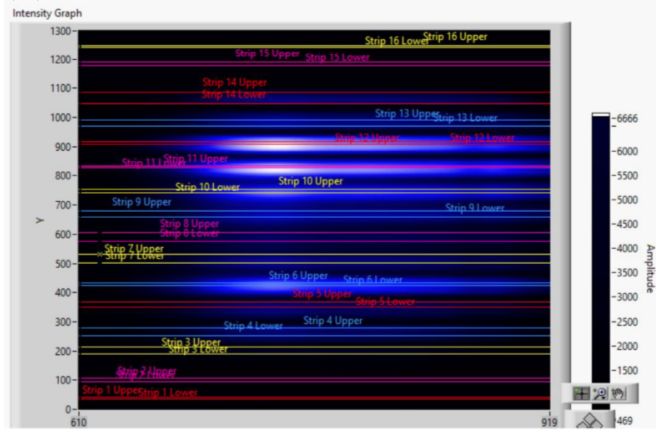

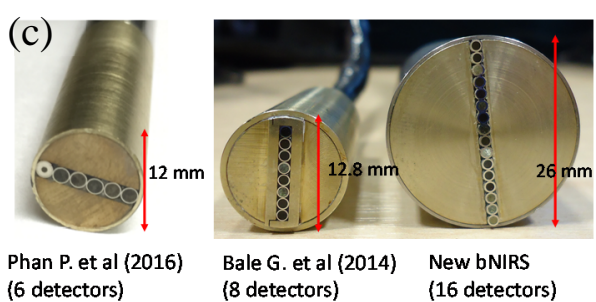

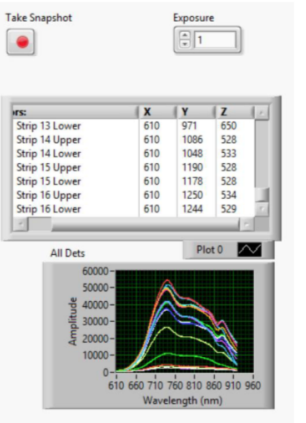

Figure 1. a) Picture of the 16 detector bNIRS system (only 14 detectors used); b) Picture of source and detector fibres on a solid phantom; c) Detector ferrule comparison from previous instruments Phan P. et al (2016) [4], Bale G. et al (2014) [3] and current instrument; (d) Image of the software acquisition illustrating the CCD binning for each detector.

\subsection{Protocol}

We studied 12 patients at Great Ormond Street Hospital (GOSH, London, UK) under ethics (17/LO/1402); 5 females and 7 males, from 23 months old to 14 years old. A custom made probe holder allowed us to measure either the frontal cortex or the left and right parietal lobes. Monitoring was done for 30minutes to 1hour during clinical assessment of the patient that in some patients included a hyperventilation task. The bNIRS probe positioned after the EEG, over the suspected region of the seizures. We present data here from a 3 year old female patient (see Figure 2) with seizure activity starting in the left posterior region. They present with fast rhythmic activity which usually evolves into generalized spikewave discharges in a few seconds.

\section{RESULTS}

The bNIRS instrument captured the seizure activity with high temporal and spatial resolution. In Figure 2 we can see in some channels (L1, L2 and R3) the presence of spikes in the haemoglobin measurements; only oxCCO in L2 demonstrates the same behavior. Channels L1 and L2 are nearest to the front were seizure activity was initiated. Average data across all 
seizures (not presented here) shown that during the seizures period, channel L2 had an increase in oxCCO while channel L4 had a decrease in oxCCO.
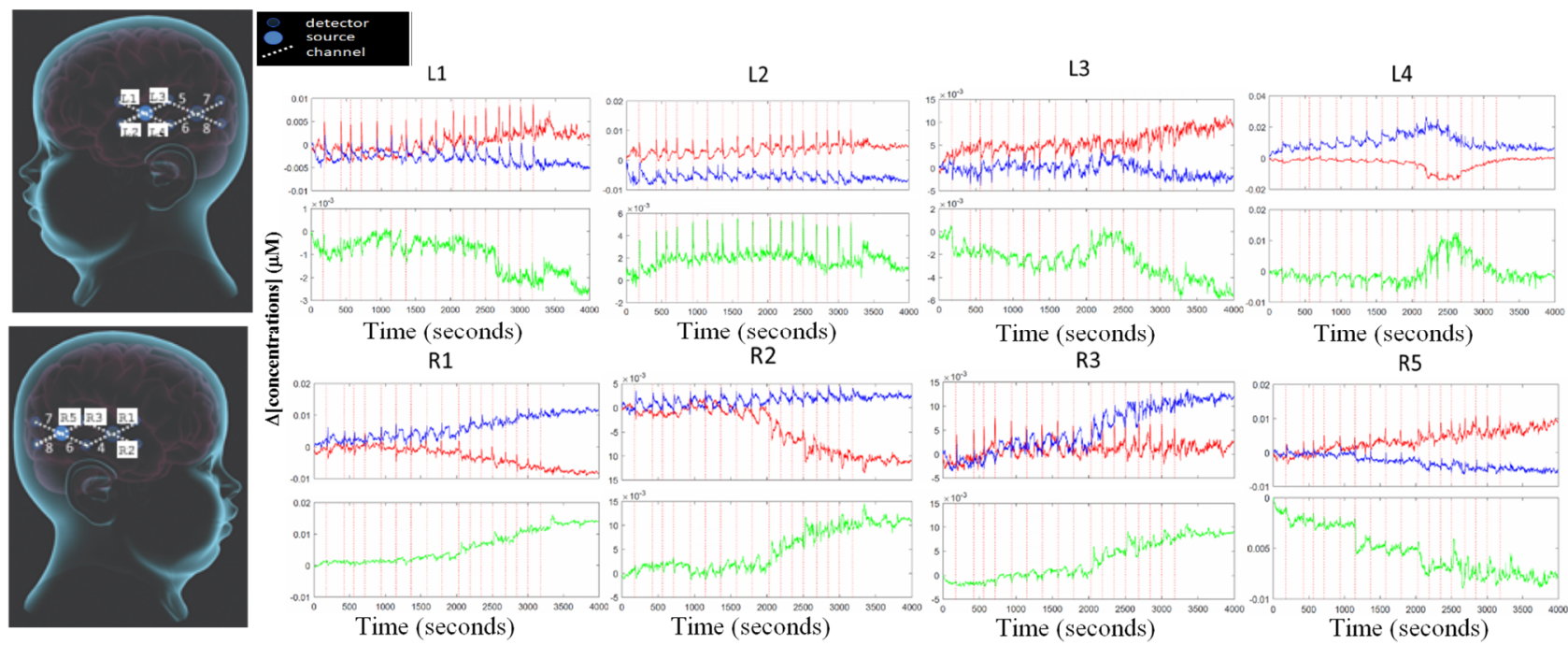

Time (seconds)

R5

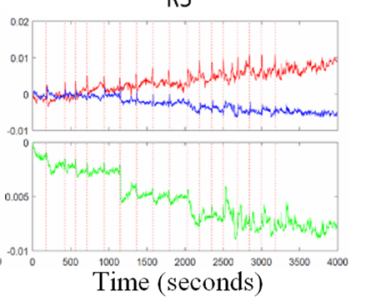

Figure 2. Changes in brain tissue concentrations of $\left[\mathrm{HbO}_{2}\right]$ (red line), [HHb] (blue line) and [oxCCO] (green line) over four channels on the left and four channels on the right side of the head. Vertical magenta lines represent the start of a seizure as identified by the EEG. L: is left side; R: is right side.

\section{DISCUSSION}

We describe a new developed bNIRS instrument with the capacity to measure both haemoglobin oxygenation and metabolism by the quantification of oxCCO over 16 locations on the head with 1 second sampling rate. We demonstrated the use of the instrument in monitoring seizures. Previously Mitra and colleagues (2016) [2] showed a rapid increase in oxCCO at the onset of seizures along with a rise in the baseline of amplitude-integrated electroencephalogram (EEG); in addition cerebral oxygenation and cerebral blood volume fell just prior to the seizure onset but recovered rapidly during seizures. In this study we observed similar trend in one of the channels where seizures have initiated. Most interestingly the right hemisphere showed an overall increase in the oxidation of CCO across the 1hour monitoring; this was not seen in the left side indicating the capacity of the right hemisphere to sustain and increase brain tissue metabolism due to the seizures activity.

\section{REFERENCES}

[1] Bale, G., Elwell, C. E. and Tachtsidis, I. "From Jöbsis to the present day: a review of clinical near-infrared spectroscopy measurements of cerebral cytochrome-c-oxidase," J. Biomed. Opt. 21(9), 091307 (2016).

[2] Mitra S, Bale G, Mathieson S, Uria-Avellanal C, Meek J, Tachtsidis I, Robertson NJ. "Changes in Cerebral Oxidative Metabolism during Neonatal Seizures Following Hypoxic-Ischemic Brain Injury.” Front Pediatr, 10(4), 83 (2016)

[3] Bale G, Mitra S, Meek J, Robertson N, Tachtsidis I. "A new broadband near-infrared spectroscopy system for invivo measurements of cerebral cytochrome-c-oxidase changes in neonatal brain injury." Biomed Opt Express, 5(10), 3450-66 (2014)

[4] Phan P, Highton D, Lai J, Smith M, Elwell C, Tachtsidis I. "Multi-channel multi-distance broadband near-infrared spectroscopy system to measure the spatial response of cellular oxygen metabolism and tissue oxygenation." Biomed Opt Express, 7(11), 4424-4440 (2016) 